\title{
Multi gene mutation signatures in colorectal cancer patients: predict for the diagnosis, pathological classification, staging and prognosis
}

Yan Zhuang ${ }^{1 \dagger}$, Hailong Wang ${ }^{2 \dagger}$, Da Jiang $^{3}$, Ying Li ${ }^{3}$, Lixia Feng ${ }^{4}$, Caijuan Tian $^{5}$, Mingyu Pu ${ }^{5}$, Xiaowei Wang ${ }^{6}$, Jiangyan Zhang ${ }^{6}$, Yuanjing $\mathrm{Hu}^{7^{*}}$ and Pengfei Liu ${ }^{2^{*}}$

\begin{abstract}
Background: Identifying gene mutation signatures will enable a better understanding for the occurrence and development of colorectal cancer (CRC), and provide some potential biomarkers for clinical practice. Currently, however, there is still few effective biomarkers for early diagnosis and prognostic judgment in CRC patients. The purpose was to identify novel mutation signatures for the diagnosis and prognosis of CRC.

Methods: Clinical information of 531 CRC patients and their sequencing data were downloaded from TCGA database (training group), and 53 clinical patients were collected and sequenced with targeted next generation sequencing (NGS) technology (validation group). The relationship between the mutation genes and the diagnosis, pathological type, stage and prognosis of CRC were compared to construct signatures for CRC, and then analyzed their relationship with RNA expression, immunocyte infiltration and tumor microenvironment (TME).

(Continued on next page)
\end{abstract}

\footnotetext{
*Correspondence: huyuanjingtianjin@163.com; 9957600@163.com

${ }^{\dagger}$ Yan Zhuang and Hailong Wang contributed equally to this work.

${ }^{7}$ Department of Gynecological Oncology, Tianjin Central Hospital of

Obstetrics \& Gynecology, No. 156 Nankai Third Road, Nankai District, Tianjin

300100, China

2Department of Oncology, Tianjin Academy of Traditional Chinese Medicine

Affiliated Hospital, No.354 Beima Road, Hongqiao District, Tianjin 300120,

China

Full list of author information is available at the end of the article
}

(c) The Author(s). 2021 Open Access This article is licensed under a Creative Commons Attribution 4.0 International License, which permits use, sharing, adaptation, distribution and reproduction in any medium or format, as long as you give appropriate credit to the original author(s) and the source, provide a link to the Creative Commons licence, and indicate if changes were made. The images or other third party material in this article are included in the article's Creative Commons licence, unless indicated otherwise in a credit line to the material. If material is not included in the article's Creative Commons licence and your intended use is not permitted by statutory regulation or exceeds the permitted use, you will need to obtain permission directly from the copyright holder. To view a copy of this licence, visit http://creativecommons.org/licenses/by/4.0/ The Creative Commons Public Domain Dedication waiver (http://creativecommons.org/publicdomain/zero/1.0/) applies to the data made available in this article, unless otherwise stated in a credit line to the data. 


\begin{abstract}
(Continued from previous page)
Results: Mutations of TP53, APC, KRAS, BRAF and ATM covered $97.55 \%$ of TCGA population and $83.02 \%$ validation patients. Moreover, $57.14 \%$ validation samples and 22.06\% TCGA samples indicated that patients with mucinous adenocarcinoma tended to have BRAF mutation, but no TP53 mutation. Mutations of TP53, PIK3CA, FAT4, FMN2 and TRRAP had a remarkable difference between I-II and III-IV stage patients $(P<0.0001)$. Besides, the combination of PIK3CA, LRPIB, FAT4 and ROS1 formed signatures for the prognosis and survival of CRC patients. The mutations of TP53, APC, KRAS, BRAF, ATM, PIK3CA, FAT4, FMN2, TRRAP, LRP1B, and ROS1 formed the signatures for predicting diagnosis and prognosis of CRC. Among them, mutation of TP53, APC, KRAS, BRAF, ATM, PIK3CA, FAT4 and TRRAP significantly reduced their RNA expression level. Stromal score, immune score and ESTIMATE score were lower in patients with TP53, APC, KRAS, PIK3CA mutation compared non-mutation patients. All the 11 gene mutations affected the distributions of immune cells.

Conclusion: This study constructed gene mutation signatures for the diagnosis, treatment and prognosis in CRC, and proved that their mutations affected RNA expression levels, TME and immunocyte infiltration. Our results put forward further insights into the genotype of CRC.
\end{abstract}

Keywords: Colorectal cancer (CRC), Genotype, Pathological classification, Staging, Prognosis

\section{Background}

Colorectal cancer (CRC) is one of the most prevalent and lethal malignant diseases worldwide. According to the latest data, the incidence and mortality of CRC ranks 3rd and 2th, respectively, among all global cancer patients [1]. Like most cancers, early diagnosis of CRC contributes to a good prognosis. The 5-year survival for persons with CRC is $64 \%$ in the United States. If the disease is detected at an early stage, the 5-year survival rate can be increased to $90 \%$ [2]. Simultaneously, pathological classification is also important indicator of prognosis. For example, mucinous colorectal adenocarcinoma is a distinct subtype of CRC, which is more frequently diagnosed in advanced stages and usually has poorer responses to chemotherapy [3]. Tumor microenvironment (TME) plays an important role in tumor development. The synergistic interaction between cancer cells and their supporting cells leads to the malignant tumor. Therefore, TME has a significant effect in cancer patients. At the same time, studies have paid close attention to the effect of immune cells in TME on tumor growth and progression, for example, tumor infiltrating immune cells (TIC) in TME can be used as a promising indicator of therapeutic effect [4]. However, there is still a lack of effective biomarkers for early diagnosis nowadays, and there are few effective prognostic judgment and evaluation indicators for CRC patients.

Genome instability promotes the accumulation of mutations in cancer cells and leads to the rapid evolution of cancer genomes in response to tumor microenvironment and treatment-induced stress. In recent years, data from a large number of genome-wide and exome-wide project have basically elucidated the mutations in most common cancers, and revealed the functional and structural characteristics of cancer genome [5]. Different mutational processes often generate different combinations of mutation types, named as "signatures" [6]. Recently, some studies indicated mutation gene signatures have good performances in predicting the treatment and prognosis in CRC patients [7]. For example, KRAS, BRAF and PIK3CA may have prognostic values in CRC [8], mutation detection of combination of KRAS, BRAF and PIK3CA could contribute to predict the response of CRC patients to EGFR pathway inhibitors [9]. Yu et al. [10] reported that the mutation status of five gene signatures, CDH10, COL6A3, SMAD4, TMEM132D and VCAN, could predict the survival of CRC patients in two independent cohorts. Ye et al. [11] found that BRAF mutation was associated with poor prognosis in Chinese patients receiving anti-EGFR therapy. Furthermore, abundant scientific researches and medical practices have been made and achieved enormous progress about some mutations of them, such as APC, TP53, KRAS, BRAF, PTEN and PIK3CA in the diagnosis, treatment and prognosis of CRC $[12,13]$. The NCCN (National Comprehensive Cancer Network) guidelines present that individual genetic test, including $K R A S$, NRAS, BRAF, etc. can be used for CRC diagnosis and treatment. However, more signatures of gene mutation are still needed to better understand the somatic mutations and genotype of CRC. In addition, studies have shown that gene mutation changes its RNA expression level and immune characteristics, but whether there is an impact in CRC remains unclear.

Incomplete understanding of the genotype is not conducive to accurate treatment. In the present study, we revealed some novel signatures for diagnosis, pathological classification, staging and prognosis of CRC, as well as their correlation with expression level, immune score and distribution of immune cells, in order to give further genetic insights into CRC and provide more comprehensive references for clinical practice. 


\section{Materials and methods \\ Cohorts and samples}

The overall study included 2 CRC cohorts, the training group $(\mathrm{T})$ and validation group $(\mathrm{V})$. The training group covered 531 patients from 2 The Cancer Genome Atlas projects (TCGAs), namely, The Cancer Genome Atlas Colon Adenocarcinoma (TCGA-COAD, $n=397$ ) and Rectal Adenocarcinoma (TCGA-READ, $n=134$ ). TCGA-COAD and TCGA-READ mainly study the genetic information of colon adenocarcinoma and rectal adenocarcinoma, respectively. The validation group contained 53 patients, which were collected in department of colorectal oncology of Tianjin Medical University Cancer Institute and Hospital from April 2014 to November 2018.

In training group, all the patients had the tumor tissues and matched normal tissues. The whole genome sequencing (WGS), clinical, and demographic data were downloaded from TCGA (https://tcga-data.nci.nih.gov/docs/ publications/tcga/). In the validation group, fresh tumor tissues were sequenced by targeted NGS of the 1000 gene panel (Supplementary Table S1), while 36 paracancerous tissues or 17 leukocytes were used as controls. All patients were followed up by telephone and electronic case, and the follow-up was up to July 20, 2019.

\section{Genomic DNA extraction}

The $\geq 3 \mathrm{~g}$ fresh tissue was collected in tube containing the preservation solution after operation. The genomic DNA was extracted with the Genomic DNA extraction kit (Qiagen, Hilden, Germany) following the manufacturer's instructions. Afterwards, the DNA samples were purified by Agencourt AMPure XP beads (Agentcourt Biosciences, Beverly, MA, USA).

\section{Library preparation and targeted next generation sequencing}

Library preparation for each sample was performed according to the manufacturer's protocol. Briefly, $\sim 1 \mu \mathrm{g}$ DNA was randomly sheared into 150-200-base pair fragments using a Covaris M220 instrument (Woburn, MA, USA), followed by library construction with a KAPA Hyper DNA Library Prep Kit (KAPA Biosystems, Wilmington, MA, USA). The adaptor library was amplified and linked, and the total library was accurately quantified by Qubit DNA HS Assay Kit (Invitrogen, CA, USA). A library hybridization kit, SeqCap EZ MedExome Enrichment kits (Roche, Basel, $\mathrm{CH}$ ), was used to capture target sequences and bead capture and elution hybridization libraries with Roche's customized 1000 targeted gene probes (Roche, Basel, $\mathrm{CH}$ ). To construct the targeted gene list, we referred to FoundationOne and Integrated Mutation Profiling of Actionable Cancer Targets (IMPACT), which were designed by two authoritative organizations, Foundation Medicine and Memorial Sloan Kettering Cancer Center (MSK), respectively, and all received FDA approval.

After amplifying the captured library by PCR, the constructed library was sequenced by an Illumina HiSeq Xten sequencer (San Diego, CA, USA). The average sequencing depth of tissue samples was $500 \mathrm{X}$. It could detect mutations with very low frequency to $0.1 \%$.

\section{Somatic variant detection}

The sequencing data were mapped to the human reference genome (hg19) with Burrows-Wheeler Aligner software for tumor-specific somatic mutation detection. MuTect version 1.1.4 was adopted to process the alignments and identify somatic mutations in tumor tissues compared with the matched normal tissues or leukocytes.

\section{Mutation signatures}

$\mathrm{T}$ test or $\mathrm{F}$ test in software $\mathrm{R}$ (version 3.4.1) was used to detect the correlation between gene variation distribution and each index (cancer species, stage, total survival period, sex, age and race), and the genes with $P \leq 0.05$ were selected. The ggplot2 (version 2.2.1) was used to display the gene variation distribution of each model.

\section{Signatures validation}

$\mathrm{R}$ (version 3.6.1) and IBM SPSS statistics (version 21) were selected for statistical analysis. T-test was used for continuous variables, chi-square test was used for categorical variables, logistic regression was used for cancer classification and staging model validation, and Cox model was used for survival related genes validation. The analysis results were shown using ggplot2 (version3.2.1).

\section{Tumor microenvironment and immunocyte infiltration}

The stromal cells and immune cells in tumor tissue were evaluated by the ESTIMATE algorithm in estimate package in $\mathrm{R}$ (version 3.6.1) [14]. The stromal score and immune score were estimated according to the specific biomarkers related to stromal cells and immunocyte infiltration in tumor samples [15]. The stromal scores, immune scores, tumor purity, and estimated scores of each sample were calculated. The higher scores represent higher proportion of corresponding components in TME. Then, the scores and the compositions of 28 kinds of immune cells were compared between different gene mutation states. The data were statistically analyzed with Mann Whitney $\mathrm{U}$ test using $\mathrm{R}$ (version 3.6.3), $P<0.05$ was statistically significant. 


\section{Results}

\section{The clinical and sample information}

Firstly, we counted the clinical and sample information of patients in the TCGA and validation group. The characteristics of TCGA samples mainly included sex, age, stage, pathological type, race and overall survival (OS) (detailed in Fig. 1a and Supplementary Table S2), which were downloaded directly from TCGA database. While for validation samples, more clinical indicators were involved, such as living habits (drinking, smoking), current medical history (heart disease, hypertension, diabetes, etc.), tumor size and differentiation degree, etc. (detailed in Table 1, Fig. 1b). However, due to race differences, epidemiological trend of diseases, patient's acceptance and will of gene detection and other reasons, some clinical characteristics of the validation samples were different from those of TCGA. The median age of the TCGA and validation group was 68 and 60, respectively. The TCGA group included 154 III (29.00\%) and 78 IV (14.69\%) patients, the validation group had 20 III (37.70\%) and 16 IV (30.20\%) patients. The cohort difference was mainly manifested in patients' age and tumor stage, and the validation group we collected included more patients < 60 years old $(45.28 \%$ vs. $29.76 \%, P=$ $0.019)$, and more III-IV patients $(67.90 \%$ vs. $43.69 \%, P=$ 0.004). The latest large-scale research data both in the
United States and Europe revealed that the incidence of CRC is increasing at $40-50$ years old, as is the data before 30 years old $[16,17]$. The reasons may be related to eating habits, obesity, early screening and so on. Like the United States and some European countries, CRC also has a younger trend in China. Unfortunately, screening in China is not as popular as in the United States and some European countries, and most patients are found to be in the late stage, worse in some rural and remote areas. Therefore, our data were consistent with the incidence trend of CRC and the actual situation in China, and it was reasonable to be used as a validation group.

\section{The gene somatic mutation landscape}

In general, we identified 211, 690 mutations, appeared in 18, 298 genes in TCGA cases, and 1, 953 mutations involving 631 genes in validation cases. Furthermore, 44 mutation genes were shared in both cohorts, and they were with frequency of more than $5 \%$. The 44 genes were TP53, APC, KRAS, BRAF, ATM, KMT2C, LRP1B, NF1, FBXW7, MTOR, PTEN, PIK3CA, RET, NTRK3, FAT1, SMARCA4, FAT4, SMAD4, ARID1A, ERBB4, BRCA2, FMN2, AXIN2, ERBB3, NOTCH3, MED12, KDR, TCF7L2, RNF43, PREX2, CTNNB1, ALK, NRAS, JAK1, KMT2D, TRRAP, CREBBP, ROS1, PDGFRA,

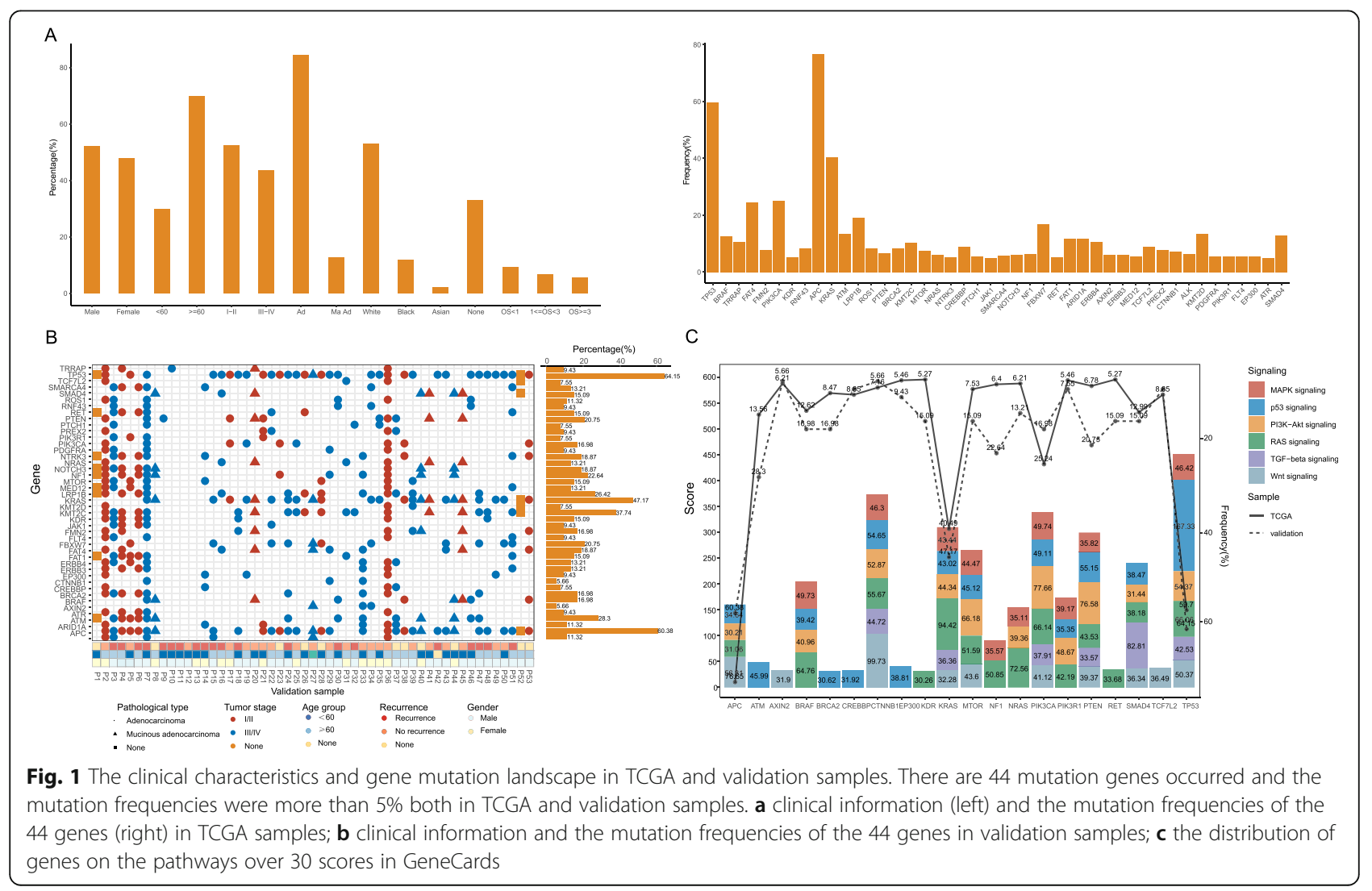


Table 1 The clinical information of validation samples

\begin{tabular}{|c|c|c|c|c|c|c|c|}
\hline Index & Classification & $\begin{array}{l}\text { Case } \\
\text { number }\end{array}$ & $\begin{array}{l}\text { Percentage } \\
\text { (\%) }\end{array}$ & Index & Classification & $\begin{array}{l}\text { Case } \\
\text { number }\end{array}$ & $\begin{array}{l}\text { Percentage } \\
\text { (\%) }\end{array}$ \\
\hline \multirow[t]{2}{*}{ Sex } & male & 37 & 69.80 & \multirow{4}{*}{$\begin{array}{l}\text { Tumor differentiation } \\
\text { status }\end{array}$} & high & 1 & 1.89 \\
\hline & female & 16 & 30.20 & & medium & 34 & 64.20 \\
\hline \multirow[t]{3}{*}{ Age } & $<60$ & 24 & 45.28 & & low & 12 & 22.61 \\
\hline & $\geq 60$ & 28 & 52.83 & & None & 6 & 11.30 \\
\hline & None & 1 & 1.90 & Hypertension & Yes & 20 & 37.70 \\
\hline \multirow[t]{3}{*}{ Tumor stage } & $|-| \mid$ & 15 & 28.30 & & No & 31 & 58.50 \\
\hline & III-IV & 36 & 67.90 & & None & 2 & 3.80 \\
\hline & None & 2 & 3.80 & Diabetes & Yes & 5 & 9.40 \\
\hline \multirow[t]{3}{*}{$\begin{array}{l}\text { Primary } \\
\text { diagnosis }\end{array}$} & $\begin{array}{l}\text { Mucinous } \\
\text { adenocarcinoma }\end{array}$ & 7 & 13.20 & & No & 46 & 86.80 \\
\hline & $\begin{array}{l}\text { Other } \\
\text { adenocarcinoma }\end{array}$ & 44 & 83.00 & & None & 2 & 3.80 \\
\hline & None & 2 & 3.80 & Coronary heart & Yes & 6 & 11.30 \\
\hline \multirow[t]{4}{*}{ KPS score } & 80 & 3 & 5.70 & & No & 45 & 84.90 \\
\hline & 90 & 23 & 43.40 & & None & 2 & 3.80 \\
\hline & 95 & 12 & 22.60 & Other diseases & Yes & 15 & 28.30 \\
\hline & None & 15 & 28.30 & & No & 36 & 67.90 \\
\hline \multirow{3}{*}{$\begin{array}{l}\text { Drinking } \\
\text { habit }\end{array}$} & Yes & 30 & 56.60 & & None & 2 & 3.80 \\
\hline & NO & 12 & 22.60 & Therapeutic method & $\begin{array}{l}\text { Neoadjuvant chemotherapy }+ \\
\text { Operation }\end{array}$ & 4 & 5.70 \\
\hline & None & 11 & 20.80 & & Operation only & 24 & 45.30 \\
\hline \multirow{3}{*}{$\begin{array}{l}\text { Smoking } \\
\text { habit }\end{array}$} & Yes & 24 & 45.30 & & Operation + chemotherapy & 23 & 43.40 \\
\hline & NO & 23 & 43.40 & & $\begin{array}{l}\text { Neoadjuvant } \\
\text { chemotherapy+Operation+Targeting }\end{array}$ & 2 & 3.80 \\
\hline & None & 6 & 11.30 & OS & $<1$ year & 17 & 32.08 \\
\hline \multirow[t]{3}{*}{ Tumor size } & $<5 \mathrm{~cm}$ & 23 & 43.40 & & $1-3$ years & 16 & 30.19 \\
\hline & $\geq 5 \mathrm{~cm}$ & 26 & 49.10 & & $\geq 3$ years & 5 & 9.43 \\
\hline & None & 4 & 7.50 & & None & 5 & 9.43 \\
\hline
\end{tabular}

OS, survival time; None indicated unclear or unknown

PTCH1, PIK3R1, FLT4, EP300 and ATR. Among them, both in the two cohorts, the top 3 most frequent genes were APC, TP53 and KRAS, and 76.65, 59.70 and $40.49 \%$ in TCGA cases, and $60.38,64.15$ and $47.15 \%$ in validation cases, respectively. $A P C$, the "gatekeeper gene" of $\mathrm{CRC}$, which plays the role of gatekeeper by inducing apoptosis, and also participates in cell migration, adhesion, transcription activation and other processes [18]. About $80 \%$ of colorectal adenomas and adenocarcinoma have $A P C$ gene deletion or inactivation mutations, and the mutation runs through the whole process of carcinogenesis. Approximately half of all CRCs show TP53 gene mutations, which appear to have little or no prognostic value for CRC patients treated by surgery alone, but are associated with worse survival for patients treated with chemotherapy [19]. It was reported $30-50 \%$ of CRC harbor KRAS mutations, and KRAS mutations in CRC have been associated with poorer survival and increased tumor aggressiveness [20]. Mutations of APC, TP53 and $K R A S$ have been proposed as a genetic model, which drives the transition from healthy colonic epithelia to $\mathrm{CRC}$ through increasingly dysplastic adenoma, and these mutations lie on alternate pathways of CRC development. In the occurrence and development of CRC, some evidences and theories have been obtained among some of them, such as FAT4, PTCH1, ROS1, PIK3R1, CREBBP, FLT4, EP300 and PDGFRA, and many unanswered and unknown questions are to be discovered [21-25]. Nevertheless, few studies have been reported on FMN2 (T: 7.91\% and V: $16.98 \%)$, TRRAP (T: $10.73 \%$ and V: 9.43\%) and ATR (T: $5.08 \%$ and V: $9.43 \%$ ) in CRC patients. Besides, most of them were on the pathway related to the development of CRC. Figure 1c demonstrated the distribution of these genes on CRC related pathways over 30 
scores in GeneCards database (https://www.genecards. org/). The scores represent the correlation between genes and pathways, and this 30 score is the median value after ranking all score values in this study.

In TCGA samples, the frequencies distribution of above 44 genes were listed in Fig. 1a. In addition to APC, TP53 and KRAS, frequencies of FAT4 (24.48\%) and PIK3CA (25.24\%) were also greater than 20\%. FAT4, a cadherin-related protein, was shown to function as a tumour suppressor in gastric cancer by modulating Wnt/ $\beta$-catenin signaling [26]. Mutations in PIK3CA play important roles in colorectal carcinogenesis, and are prognosis biomarkers [27]. Overall, these high frequency mutations are the hot spots in CRC. In each validation case, the mutation landscape of them was exhibited in Fig. 1b. Unlike in TCGA case, TP53 was the gene with the highest mutation frequency, but not APC. Following $A P C$ and KRAS, KMT2C, ATM, LRP1B, NF1, PTEN and $F B X W 7$ were ranked in the 4th to 9th places with $>20 \%$ mutation frequency. Besides, we found that the mutation frequencies of some genes were very different between the two groups, namely, PTEN $(6.78 \%$ vs. $20.75 \%, P=$ $0.0004), A P C(76.65 \%$ vs. $60.38 \%, P=0.009), A T M$ ( $13.56 \%$ vs. $28.30 \%, P=0.004), K D R(5.27 \%$ vs. $15.09 \%$, $P=0.005)$, and $F M N 2(7.90 \%$ vs. $16.98 \%, P=0.026)$. This difference may be due to sample size, age, tumor stage, ethnicity, etc.

\section{The mutation gene signatures for the diagnosis of CRC}

According to the distribution of gene variation in the population, five genes that could cover the largest population were screened, namely, TP53, APC, KRAS, BRAF and ATM. Mutations of them covered 97.55\% (513/518) of TCGA population and $83.02 \%$ (44/53) validation patients (Fig. 2a), and can be used to distinguish between cancer and paracancer. Especially when the amount of tissue obtained is small and the pathology is difficult to judge, the signatures can be used to assist diagnosis.

In TCGA cohort, 642 APC mutations were detected in 407 cases, including 353 (54.73\%) nonsense mutations, 140 frame-shift-del (21.71\%), 74 (11.47\%) frame-shiftins, 60 (9.30\%) missense, 15 (2.33\%) splice-site; 329 TP53 mutations were detected in 317 cases, including 235 (71.43\%) missense mutations, 39 (11.85\%) nonsense, 19 frame-shift-del, 14 frame-shift-ins, 15 splice-site, 2 splice-region, 4 in-frame-del and 1 in-frame-ins; 221 KRAS mutations were detected in 216 cases, and 219 (99.10\%) were missense mutations; 70 BRAF mutations were detected in 67 cases, and 87.14\% were missense mutations; 104 ATM mutations were detected in 73 cases, and 56.73\% were missense mutations (Fig. 2a). The Venn graph was showed in Fig. $2 b$ with an online tool (http://bioinformatics.psb.ugent.be/webtools/Venn/). In detail, $27.61 \%(143 / 518)$ of cases were found to contain mutations concurrently in APC and TP53, 12.16\% (63/518) of cases were found to contain mutations concurrently in APC and KRAS, 17.57\% (91/518) mutated in APC, TP53 and KRAS in unison, $0.19 \%(1 / 518)$ mutated in APC, TP53, $K R A S$ and $B R A F$, and $0.77 \%(4 / 518)$ mutated in $A P C$, TP53, KRAS and ATM.

In validation cohort, 34 cases occurred 35 mutations in TP53, and 24 (68.57\%) were missenses; 31 cases had 43 mutations in $A P C$, and 17 frame-shift, 9 missenses and 13 nonsense; 25 cases appeared $26 \mathrm{mu}-$ tations in KRAS, and 25 (96.15\%) were missenses; 9 cases emerged 9 mutations in BRAF, and all of them were missenses; 14 cases arose 20 mutations in $A T M$, and $12(60 \%)$ were missenses (Fig. 2a). The Venn graph was showed in Fig. 2c, and the most common combination of mutations was APC and TP53 $(20.45 \%, 9 / 44)$, the combination of APC, TP53 and KRAS, as well. Next was combinations of TP53 and KRAS $(11.36 \%, 5 / 44)$, and APC and ATM (9.09\%, 4/ 44). The consistency of the combined mutation trend of APC and TP53 in the validation group and TCGA indicated that our verification results were reliable.

Although a gene or a mutation form was different in above two cohorts, mutations in TP53, APC, KRAS, $B R A F$ and $A T M$ overlapped the most patients and could be used as a diagnostic signature.

\section{The mutation gene signatures for the pathological classification of CRC}

More than $95 \%$ of CRC was adenocarcinoma, and mucinous adenocarcinoma accounts for about $10 \%$ of all cases with a poor outcome [28]. In TCGA group, 68 $(12.80 \%)$ cases were mucinous adenocarcinoma, and 448 $(84.37 \%)$ were other adenocarcinoma. There were 189 (35.59\%) right colon cancer cases and 247 (46.52\%) left colon cancer cases. In validation cohort, there were 51 (96.23\%) adenocarcinoma, including 7 mucinous adenocarcinoma and accounting for $13.21 \%$ in all cases. We found that mutations of TP53 and BRAF were significantly related to the pathological types of cancer in both cohorts (Fig. 3, $P<0.0001$ and $=0.0004$, respectively).

In TCGA cases, $54.41 \%$ mucinous adenocarcinoma cases were non-mutated both in TP53 (-) and BRAF $(-)$, and $61.16 \%$ other adenocarcinoma cases were TP53 mutated $(+)$ and BRAF (-) (Fig. 3a and c). Besides, the group of TP53 (+) and BRAF (+) tended to mucinous adenocarcinoma, as well as group of TP53 $(-)$ and BRAF (+) (Fig. 3a and c). Our research found that regardless of TP53 status, BRAF mutations were more common in mucinous adenocarcinoma. This finding was consistent with previous conclusions.

In validation cases, $59.09 \%$ other adenocarcinoma were TP53 (+) and BRAF (-), which was similar to those in TCGA cases (Fig. 3b and c). However, unlike results in 


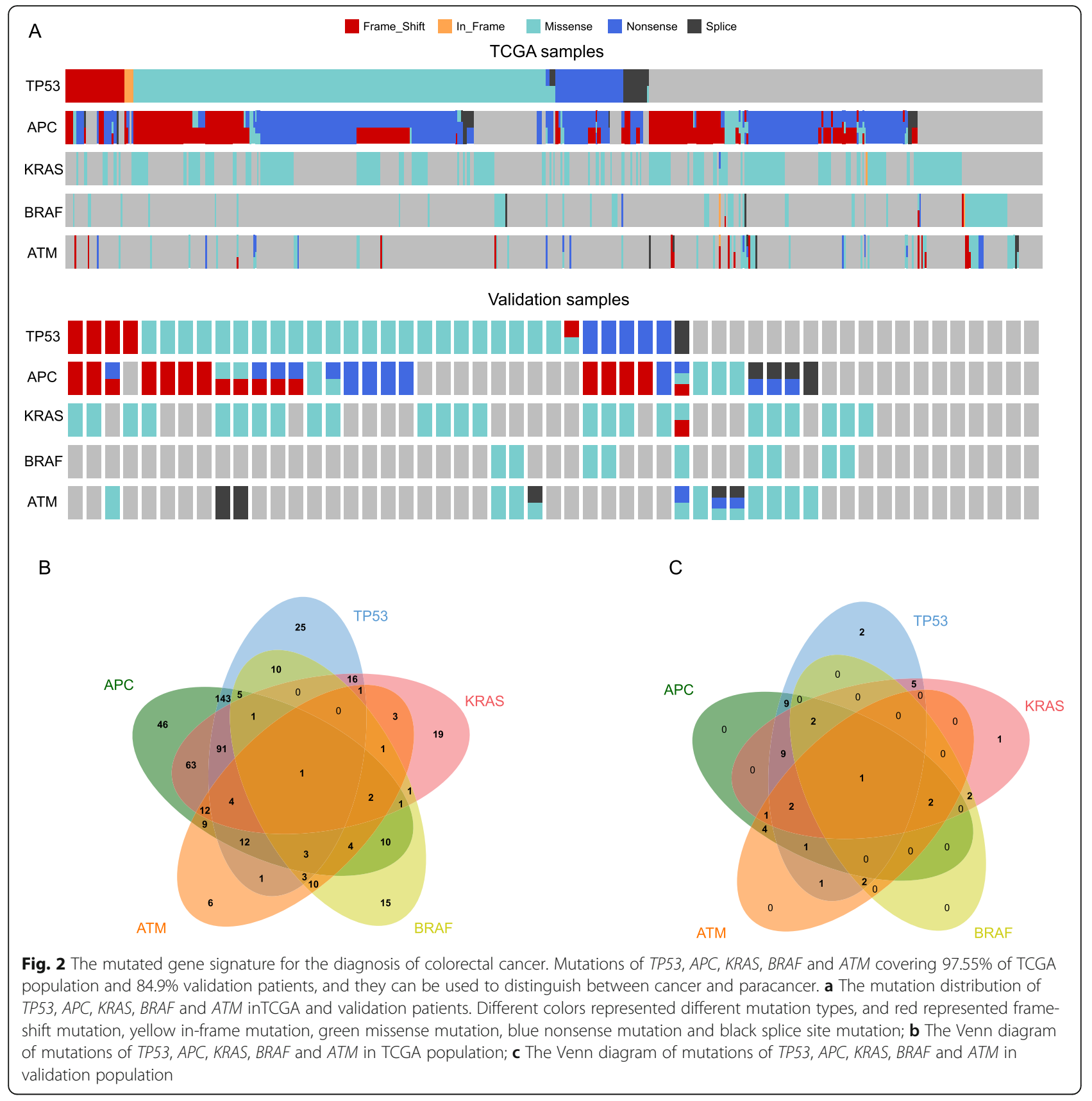

TCGA cases, all TP53 (-) and BRAF (+) cases were mucinous adenocarcinoma, and all TP53 (+) and BRAF (+) cases were other adenocarcinoma, and TP53 (-) and BRAF (-) cases had no obvious difference in different pathological types (Fig. $3 \mathrm{~b}$ and c). The reasons for the above differences may be related to the different race, age and stage distributions between the two groups, and the small number of samples in the validation group may also be one of reasons. In addition, we used logistic regression analysis in validation cases to get the coefficient of the pathological classification = $3.192 \times$ (mutation of TP53) - 2.954 $\times$ (mutation of
$B R A F)+1.493$, and mutation of TP53 or $B R A F=0$ (mutated) or 1 (no mutated); and mucinous adenocarcinoma (the coefficient $=0$ ) and other adenocarcinoma (the coefficient $=1$ ). The accuracy rate of this model was $94.1 \%$ for the whole, $100 \%$ for other adenocarcinoma, and $57.1 \%$ for mucinous type. In summary, mutation detection of TP53 and BRAF can be used for pathological classification of CRC.

The mutation gene signatures for the tumor stage of CRC Tumor staging is an important diagnostic index, which is contribute to guide treatment and prompt prognosis. 


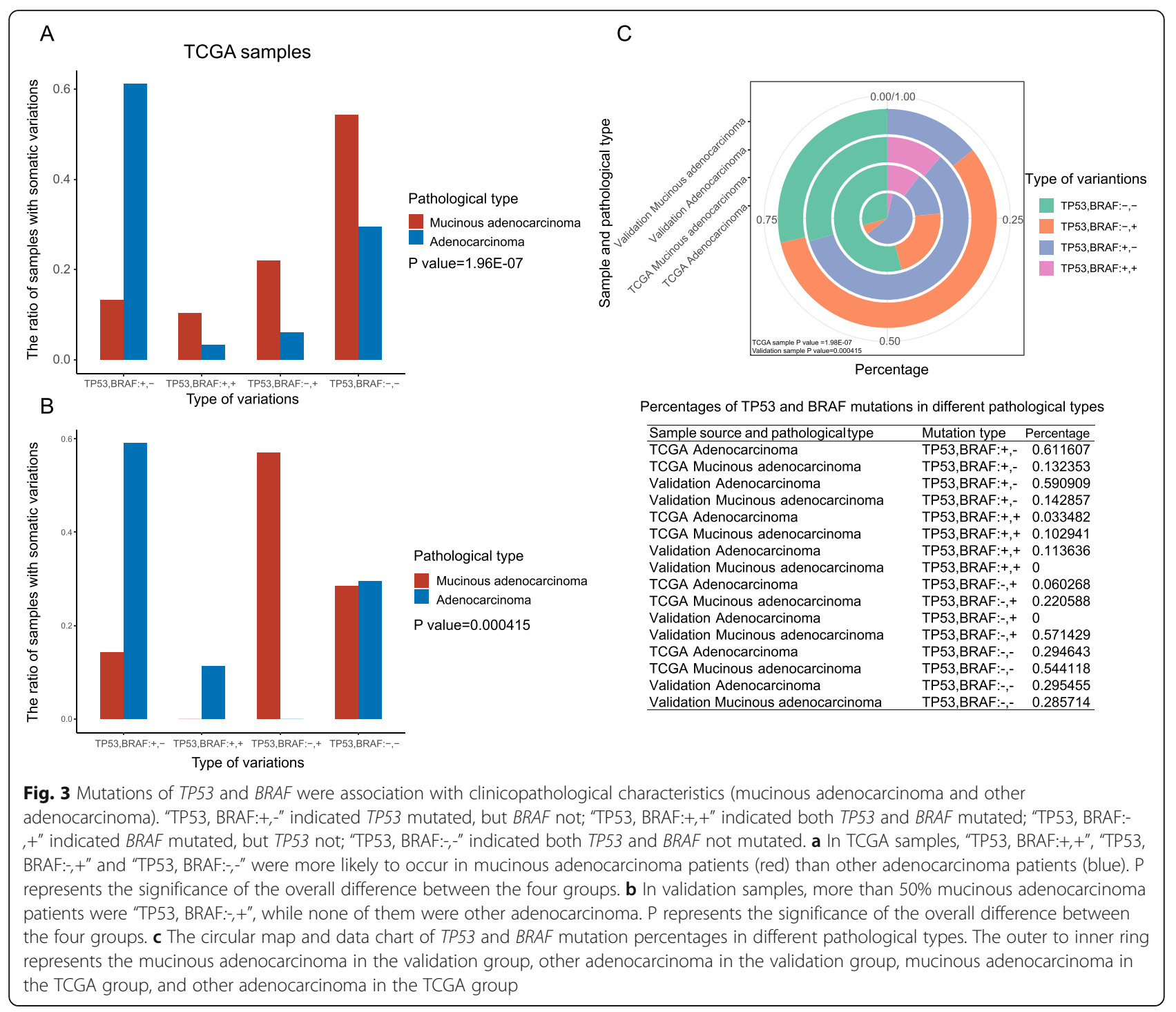

Here, we divided the samples into stage I-II and stage III-IV groups. In the discovery queue, after counting all possible combinations, the signature of 5 genes was obtained which was closely related to the tumor stage with the minimum $P$ value, namely TP53, PIK3CA, FAT4, FMN2 and TRRAP $(\mathrm{F}=18.86$ and $P<0.0001$, Fig. 4a, b). It was further confirmed in the validation queue $(P=$ 0.018 , Fig. $4 \mathrm{~b})$. Moreover, both in the discovery and validation queues, PIK3CA, FAT4, FMN2 and TRRAP had higher mutation frequencies in I-II stage group (Fig. 4b). Interestingly, TP53 mutation was contradictory between two cohorts in different stages, with higher frequency in stage III-IV group in discovery queue, and no remarkable difference between stage I-II and stage III-IV groups in validation queue (Fig. 4b). Besides, the logistic regression analysis was also performed to obtain the coefficient of the stage $=0.003 \times($ mutation of TP53) $1.937 \times$ (mutation of PIK3CA) $-0.468 \times$ (mutation of
FAT4) $-1.245 \times($ mutation of FMN2) $-1.573 \times$ (mutation of TRRAP) +1.790: mutation of gene $=0$ (mutated) or 1 (no mutated); and I or II stage (the coefficient $=0$ ) and III or IV (the coefficient $=1$ ). The accuracy rate of the mutation signature composed of TP53, PIK3CA, FAT4, FMN2 and TRRAP was $80.4 \%$ overall, $53.3 \%$ for I-II stage, and $91.7 \%$ for III-IV stage. Therefore, mutation detection of TP53, PIK3CA, FAT4, FMN2 and TRRAP could help to identify the CRC stage.

\section{The mutation gene signatures for the survival of CRC}

In the discovery cohort, samples were firstly divided into three groups according to the OS $(<1$ year, $n=50 ; 1-3$ years, $n=35$; and $>3$ years, $n=29$ ). After multiple combinations of the above 44 genes, we found that the mutation signature of PIK3CA (25.24\%), LRP1B (19.21\%), FAT4 (24.48\%) and ROS1 (8.29\%) showed the most significant difference among the above three groups $(\mathrm{F}=$ 


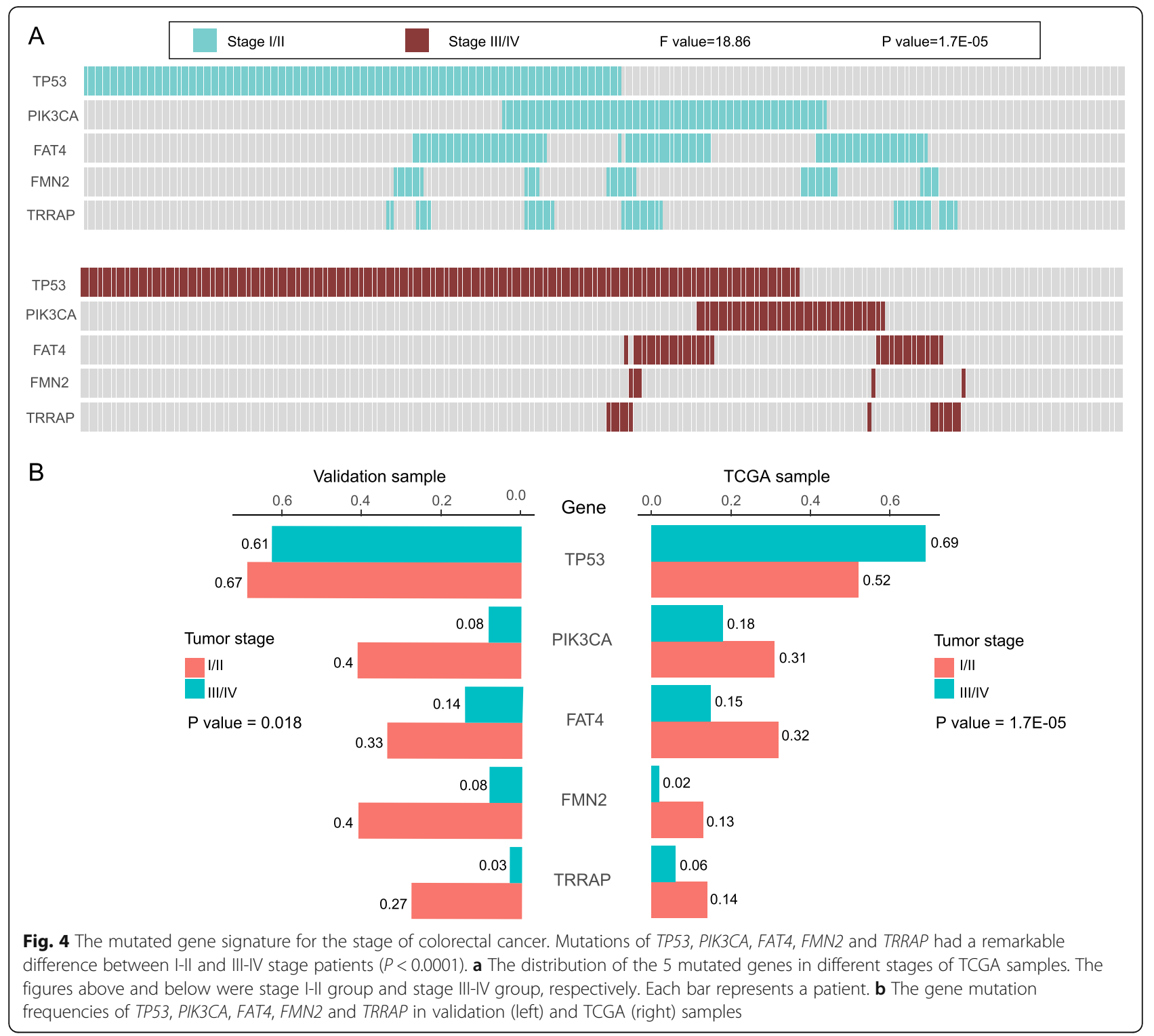

13.74 and $P=0.0003$, Fig. 5a). In our clinical samples, the above result was not authenticated. Yet, we found some other interesting results: mutation of $L R P 1 B$ portended to a higher of recurrence and shorter progression-free survival (PFS); mutation of FAT4 portended to a lower of recurrence and longer PFS (Fig. 5b). Therefore, we can detect the mutation of PIK3CA, $L R P 1 B, F A T 4$ and ROS to predict the survival of CRC patients.

Taken together, we screened 11 genes (TP53, APC, KRAS, BRAF, ATM, PIK3CA, FAT4, FMN2, TRRAP, $L R P 1 B$ and $R O S 1$ ) associated with diagnose, pathological classification, tumor stage and survival of CRC, and mutation detection of these genes can be applicable in the clinic of CRC.

\section{Correlation analysis between the CRC-related gene} mutations and RNA expression levels in the TCGA cohort

To identify the effect of the CRC-related gene mutations above on gene expressions, we conducted the correlation analysis between the mutation data and expression data in each screened gene above from the TCGA cohort. Among the 11 screened genes (TP53, APC, KRAS, BRAF, ATM, PIK3CA, FAT4, FMN2, TRRAP, LRP1B and ROS1), the mutation of 8 genes (except FMN2, LRP1B and ROS1) significantly down-regulated their own expression level $(P<0.05$, Fig. 6). Therefore, this preliminary result proved that the mutations of TP53, APC, KRAS, BRAF, ATM, PIK3CA, FAT4 and TRRAP functioned as negative factors to affect their transcriptions. 

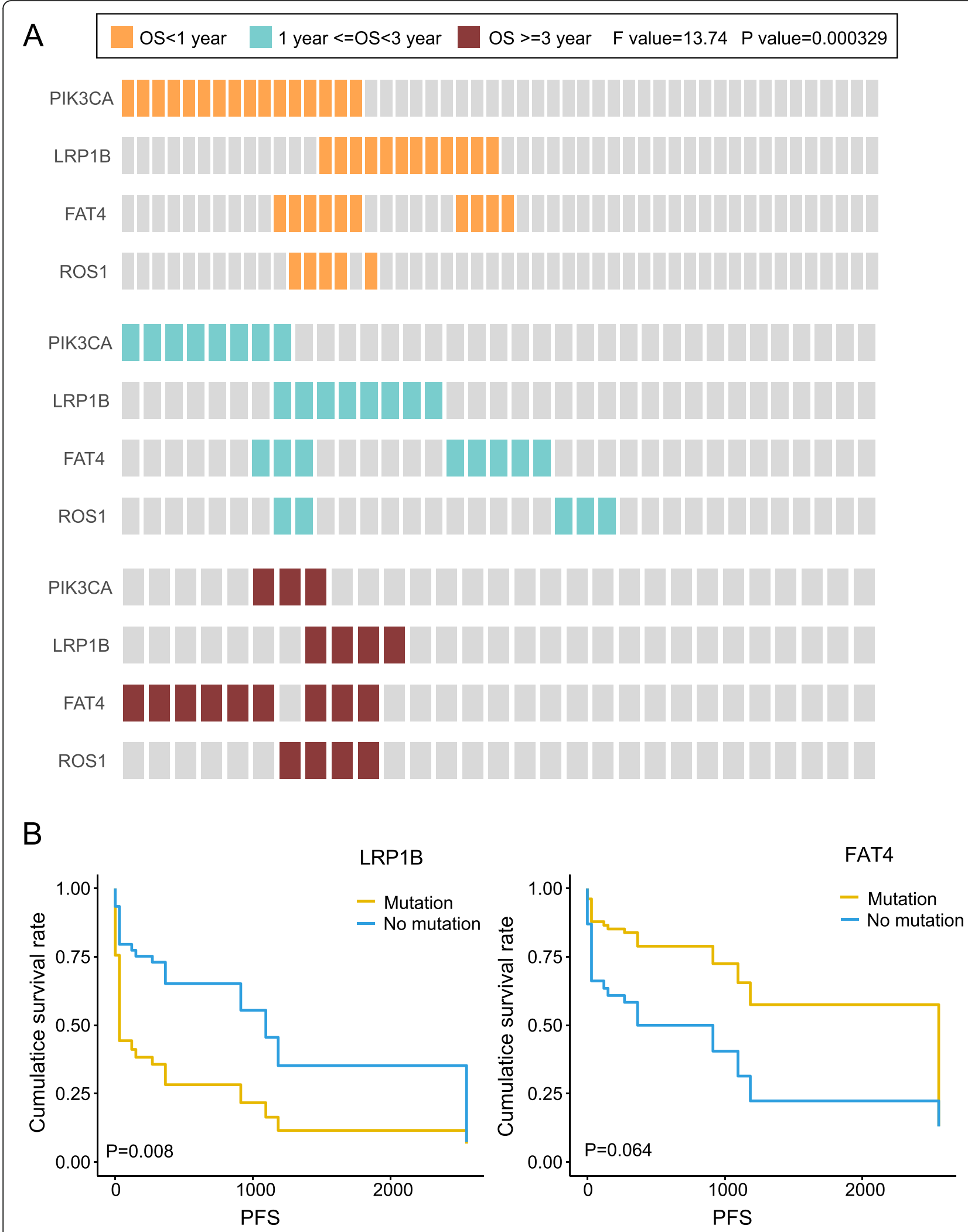

Fig. 5 (See legend on next page.) 
(See figure on previous page.)

Fig. 5 The mutated gene signature for the prognosis of colorectal cancer. PIK3CA, LRP1B, FAT4 and ROS1 were significantly correlated with overall survival $(P<0.001)$. a In TCGA samples, the distribution of the 4 mutated genes in patients with different OS. The figures from top to bottom were OS $<1$ year group, $1 \leq$ OS $<3$ years group, and OS $\geq 3$ years group in sequence. $\mathbf{b}$ In validation samples, mutation survival curve of $L R P 1 B$ (left) and FAT4 (right) genes

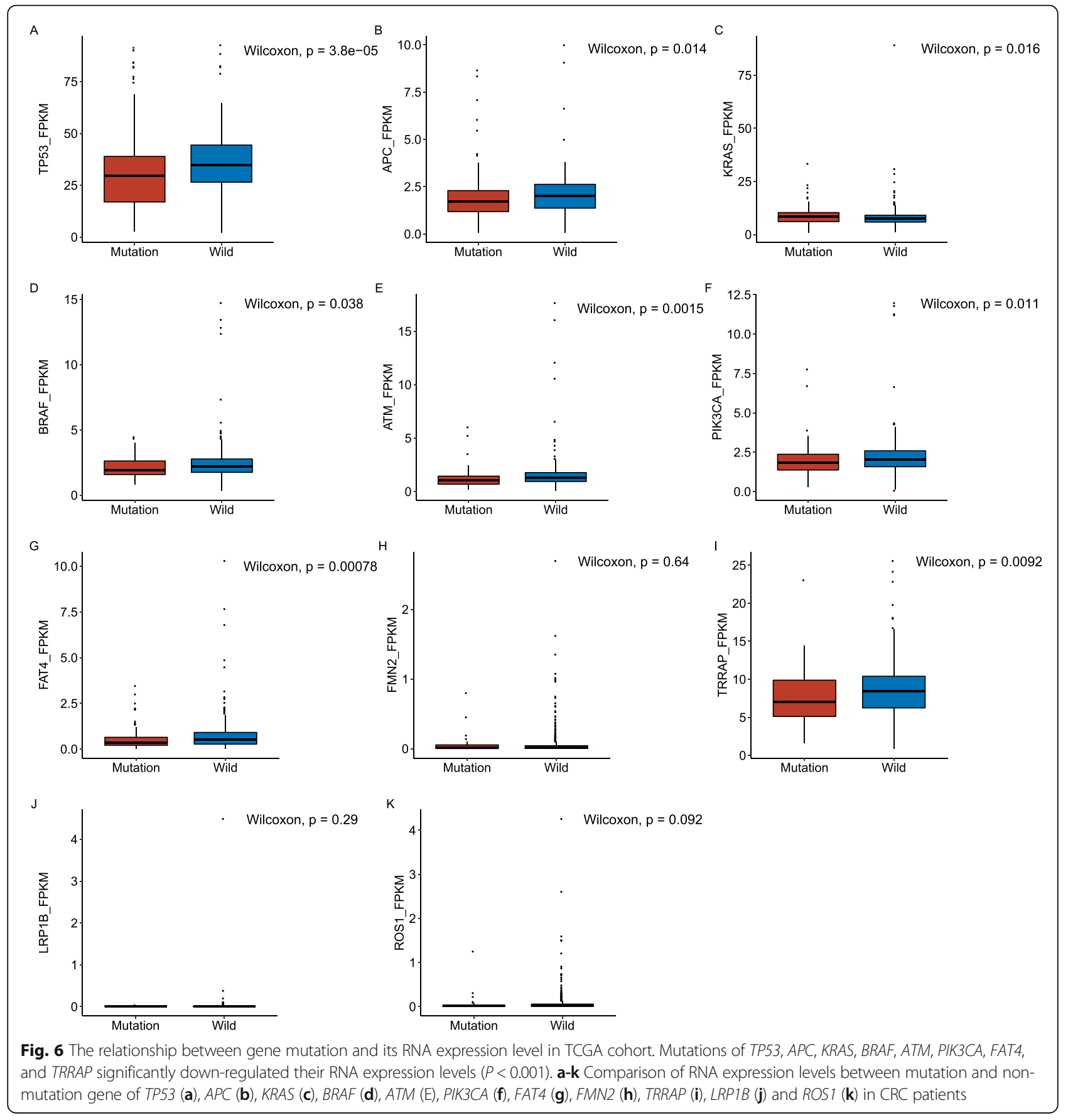




\section{Correlation analysis between the CRC-related gene mutations and immune microenvironment}

Crosstalk between cancer cells and immune microenvironment is essential to the tumor development. To identify whether the screened genes in CRC involved the immune microenvironment, we conducted a correlative analysis between the CRC-related gene mutations and immune microenvironment. Based on the gene expression data from the TCGA cohort, we calculated the abundance of 28 immune cells in the TME. Through the correlation analysis, we found that most of the cells had significant differences between the mutated and nonmutated states of the 11 screened genes (Fig. 7). Among them, activated CD4 $\mathrm{T}$ cell and T-follicular helper cell had significant differences in all genes between mutated and non-mutated status $(P<0.05)$, while the distribution of CD56dim natural killer cell, monocyte and eosinophil were different in less genes (Fig. 7). TME includes stromal cells, tumor cells, and immune cells. The higher the stromal score and immune score, the lower the purity of tumor. Figure 8 showed the TME in CRC patients between mutation and non-mutation genes. Among all genes, stromal score, immune score, and estimate core of mutant TP53, APC, KRAS, PIK3CA were lower (Fig. 8a-c), and tumor purity was relatively high (Fig. 8d). In contrast, mutant BRAF, ATM, LRP1B, FAT4, FMN2, TRRAP, and ROS1 had higher stromal score (except ATM), immune score and ESTIMATE score, and the tumor purity was lower than the wild-type (Fig. 8).

\section{Discussion}

In this report, we have identified numerous somatic mutations of CRC both in TCGA and validation cohorts, and then extended four mutation signatures for the diagnosis, pathological classification, tumor stage, and survival time, including 11 screened genes in total. These data improved the current understanding of the molecular type of CRC and facilitated clinical molecular diagnosis and prognosis prediction. Mutations of TP53, APC, $K R A S, B R A F$ and $A T M$ were a diagnosis signature for CRC; TP53 and BRAF, a mutation signature for the pathological classification; TP53, PIK3CA, FAT4, FMN2 and TRRAP, a mutation signature for tumor stage; PIK3CA, LRP1B, FAT4 and ROS1, a mutation signature for prognosis.

First and foremost, 44 shared mutated genes were identified in both cohorts with frequency of more than $5 \%$. Most of them have been studied in CRC and

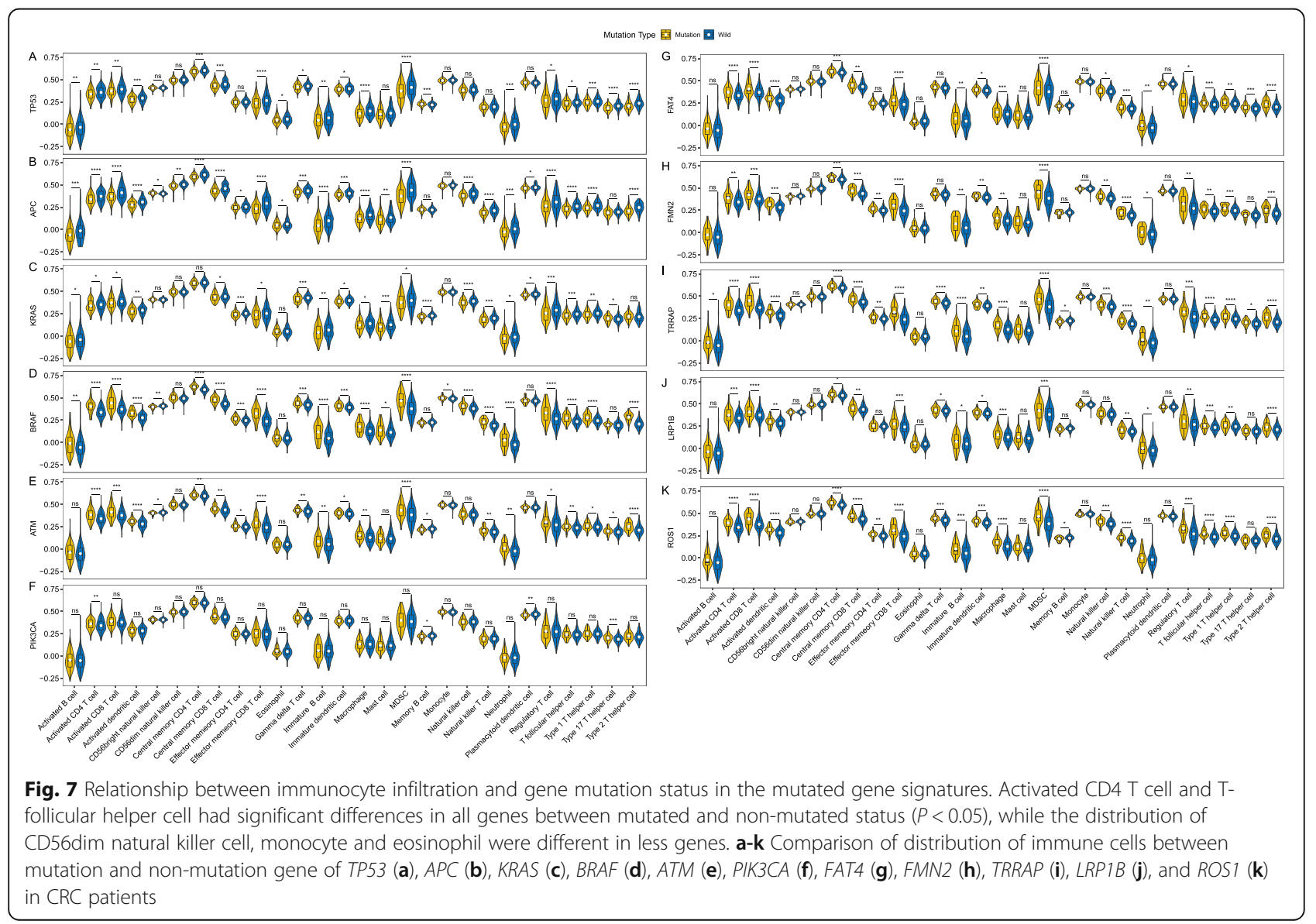




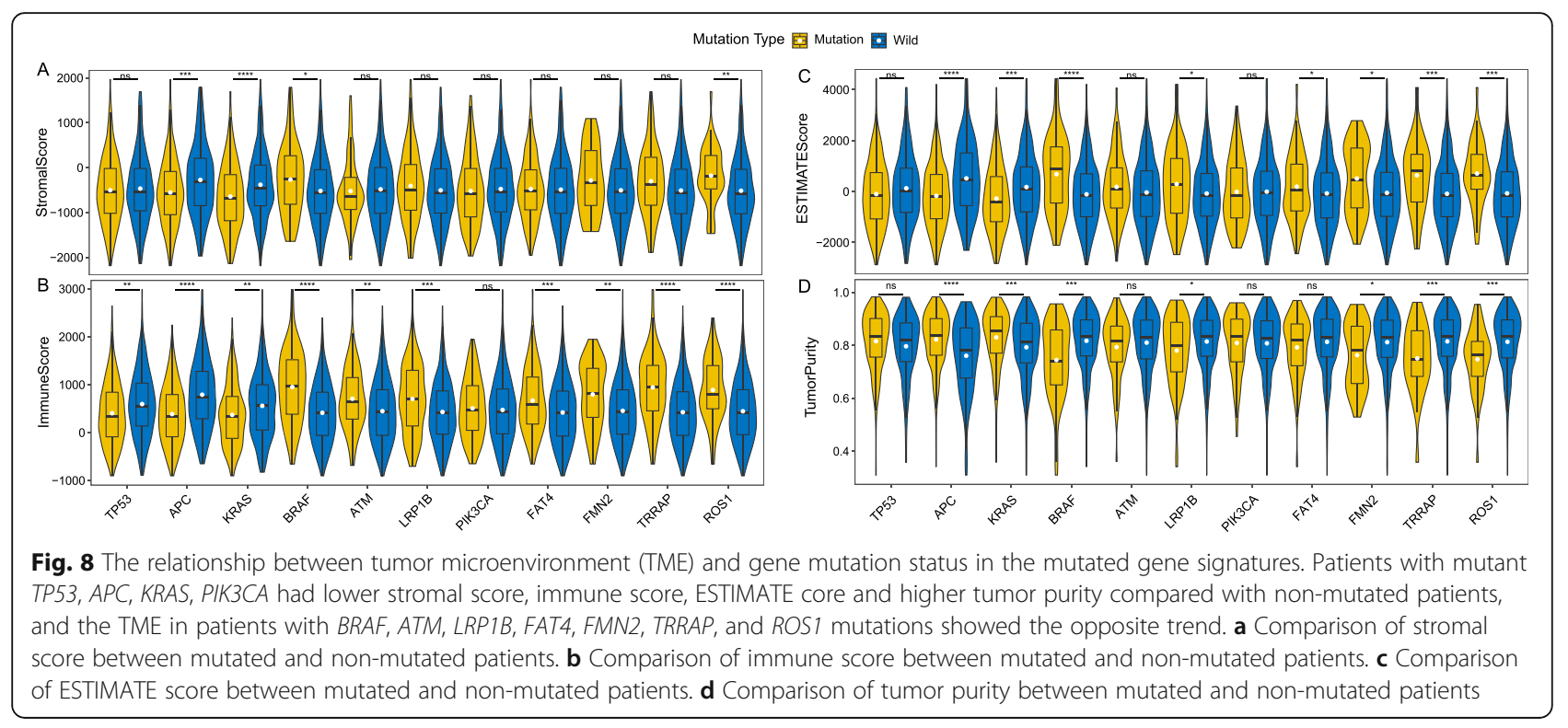

possessed similar frequencies in the 2 cohorts, suggesting these mutation genes are reliable. More specifically, it is basically formed a consensus that CRC progression is sequential gains of mutations in Wnt signaling, RAS signaling, TGF-beta signaling, p53 signaling, PI3K-Akt signaling and MAPK signaling [29]. After annotation with GeneCards database, many of them were found to be involved in the pathways related to CRC (Fig. 1c, Supplementary Fig. 1 and Table S3): CTNNB1, APC, TP53, MTOR, PIK3CA and PTEN, etc. were enriched in Wnt signaling; KRAS, NRAS, PIK3CA, TP53, BRAF and CTNNB1, etc. in RAS signaling; SMAD4, CTNNB1, TP53, PIK3CA, KRAS and PTEN in TGF-beta signaling; TP53, PTEN, CTNNB1, PIK3CA, ATM and MTOR, etc. in p53 signaling; PIK3CA, PTEN, MTOR, TP53, CTNNB1 and PIK3R1, etc. in PI3K-Akt signaling; PIK3CA, BRAF, TP53, CTNNB1, MTOR and KRAS, etc. in MAPK signaling. Additionally, PIK3CA, KRAS, CTNNB1, TP53 and PTEN are simultaneously in all of the 6 pathways listed above. This result further indicated that the 44 mutated genes we screened were meaningful and worthy of further study. This result demonstrated the importance of gene detection for high frequency mutated genes and cross genes in various pathways related to CRC from another level.

Next, we firstly discovered the mutation combination of TP53 (T: $59.70 \%$ and V: $64.15 \%$ ), APC (T: $76.65 \%$ and $\mathrm{V}: 60.38 \%$ ), KRAS (T: $40.49 \%$ and V: 47.17\%), BRAF (T: $12.62 \%$ and V: $16.98 \%$ ) and $A T M$ (T: $13.56 \%$ and V: $28.30 \%$ ) can overlap the most patients, $97.55 \%$ of TCGA population and $84.9 \%$ validation patients (Fig. 2a). A similar study reported a biochip assaying 28 mutations in the KRAS, BRAF, TP53, and APC genes for detection of $\mathrm{CRC}$, and $71 \%$ cancerous tissues were covered [30].
However, the forerunner's study did not brought ATM into and had a lower coverage. As discussed in the foregoing, TP53, APC, KRAS were the most common and popular genes, and were prone to mutation in CRC. A previous study about it reported that the progressive acquisition of activating or loss of them drives the adenoma to carcinoma transition. They also revealed that the most common combination of mutations was TP53 and $A P C(27.1 \%)$, and only $6.6 \%$ concurrently contained mutations of TP53, APC, and KRAS [31]. In our study, TP53 and APC were confirmed again to be the most common mutation combination with 27.61 and $20.45 \%$ in TCGA and validation cohorts, respectively. Whereas, the frequency of mutations of TP53, APC, KRAS in union was much higher than the previous study, $17.57 \%$ in TCGA cases, and even higher in validation cases $(20.45 \%)$. The reason for this difference may be related to the race and disease history of the enrolled patients. The previous patients were white and had no history of previous cancer or diverticular disease; while the TCGA patients contained black and Asian and the validation patients were Asian, and both of them had no restrictions on the history of disease. As for BRAF, about $10 \%$ of CRC patients are characterized by a mutation in $B R A F$ gene resulting in a valine-to-glutamate change at the residue 600 (V600E) [32]. BRAF inhibitors have been developed and benefited melanoma patients. Although, CRC patients do not respond much efficiently, and most patients at the end of the track ultimately developed resistance to these inhibitors. However, in the phase III BEACON CRC trial, encorafenib (BRAF inhibitor), binimetinib (MEK inhibitor), and cetuximab (anti-EGFR monoclonal antibody) significantly improved the OS and ORR of patients with BRAF V600E mutant mCRC 
compared to the current standard chemotherapy, and its safety is consistent with the known safety of each drug [33]. Moreover, BRAF-mutated tumors are often right sided, more recurrent in woman, higher grade, and associated with microsatellite instability (MSI) and old age [34]. In our results, the $B R A F$-mutated frequencies were separately 12.62 and $16.98 \%$ in TCGA and validation cohort, and higher in the elderly and less in mucinous adenocarcinoma $(P<0.05)$. Less in-depth researches have made about ATM mutation, and it may be associated with PARP inhibitors and EGFR-targeted therapies [35, 36]. Thus, few studies have brought ATM mutation into CRC signatures. In present study, we found the mutation ATM (T: $13.56 \%$ and V: $28.30 \%$ ) made the mutation signature of TP53 (T: $59.70 \%$ and V: $64.15 \%$ ), APC (T: $76.65 \%$ and $\mathrm{V}:$ 60.38\%), KRAS (T: $40.49 \%$ and $\mathrm{V}$ : 47.17\%), BRAF (T: $12.62 \%$ and V: $16.98 \%)$ more extensive and coverage than the other genes among the other 39 genes.

The pathological classification and tumor stage are essential indexes of the clinical diagnosis, and important references for therapeutic schedule, and they also can predict the prognosis to some extent. Hence, understanding their relationship with gene mutation may contribute to better prognosis and treatment of the disease. It was reported that $K R A S$ gene was often mutated in colorectal adenocarcinoma, and $88.9 \%$ (8/9) of KRAS mutated cases were stage III or IV diseases [37]. We did not find a correlation between KRAS mutations and adenocarcinoma, probably because the patients we enrolled were basically adenocarcinoma, except for a few unclear ones. Our data also did not find that KRAS mutation was related to III-IV stage, which may be related to the difference stages of recruitment. Patients in III-IV stage of the previous studies were less $(7.14 \%, 9 / 126)$, and those were many more in our study (70.59 and $43.69 \%$ in validation and TCGA cases). Thus, our results were more credible. Colorectal mucinous adenocarcinoma is a subtype of CRC with prominent mucin production associated with advanced stage at diagnosis, and $B R A F$ mutation [38]. In our study, we also proved mucinous adenocarcinoma tended to have BRAF mutation. More importantly, we further revealed mucinous adenocarcinoma and other adenocarcinoma were also related to the TP53 mutation, and mutations of TP53 and BRAF were considered as a signature to distinguish pathological types, which greatly complements and refines the previous studies (detailed in Fig. 3). In the same way, we firstly identified TP53, PIK3CA, FAT4, FMN2 and TRRAP as a mutation signature for tumor stage (Fig. 4). As expounded in the previous article, mutations of TP53, PIK3CA, FAT4 have a relatively clear impact and relevance on tumor staging $[19,26,27]$. However, few reports were conducted about FMN2 and TRRAP in
CRC. Here we firstly incorporated them into the tumor stage, and the signature composed of TP53, PIK3CA, FAT4, FMN2 and TRRAP had the accuracy rate of $80.4 \%$ overall, and $91.7 \%$ for III-IV stage. Besides, we used the logistic regression model to analyze the above two signatures and got the regression model. According to the model formula, we can better understand the positivenegative role and weight of each gene in the signature.

The prognosis of CRC is also an important topic in clinical and academic research. Survival time, recurrence, metastasis, and death are common prognostic factors. In TCGA cohort, we selected PIK3CA, LRP1B, FAT4 and ROS1 as a mutation signature for prognosis (Fig. 5a). At present, the relevant research is mainly focused on the single or two gene combination mutations for the study of prognosis, multiple gene combined mutations were not found in the study of prognosis. Pietrantonio F et al. [39] excavated ALK, ROS1, and NTRK rearrangements define a new and rare subtype of $\mathrm{mCRC}$ with extremely poor prognosis. Compared it, the signature of our result was with wider application. It is well known PIK3CA mutation is predictive of poor survival in patients with CRC [40]. Less evidences have been obtained to support mutation of $L R P 1 B$ and FAT4 are associated with the prognosis of CRC. Interestingly, we found mutation of $L R P 1 B$ portended to a higher recurrence and shorter PFS; mutation of FAT4 portended to a lower recurrence and longer PFS (Fig. 5b). Unfortunately, the predicted survival effect of the signature was not fully verified in the validation group. It is suspected that the signature needs further research and confirmation, which is what we will do in the future.

For the above 11 mutated genes that significantly associated with diagnosis, pathological type, stage and survival, we further analyzed the relationship between the mutation status and RNA expression level, immune invasion and TME. With the development of multi-omics analysis, much more attentions have been paid to the study of mutation effects, such as the expression of downstream genes of candidate mutations in regulatory networks, the relationship between gene mutations and changes of RNA expression levels, or the impact of mutations on pathways. Previous studies have shown that some mutations introduce stop codons prematurely and reduce mRNA transcripts, and some of them affect protein activity by changing amino acid sequences [41]. In this study, mutations of TP53, APC, KRAS, BRAF, ATM, PIK3CA, FAT4 and TRRAP resulted in a significant decrease in their RNA expression level in TCGA cohort $(P<0.05)$. However, due to the lack of transcriptome sequencing data in clinical cohort, the results are unable to verify, and the mechanism of these mutations down regulating RNA expression level and its impact on CRC were not clear. Therefore, large prospective experiments 
should be conducted in the future to investigate the mechanism of how mutations work.

The distribution of immune cells, stromal scores, immune scores and ESTIMATE scores were significantly different between wild-type and mutant of the 11 genes, and were used to verify the accuracy of these mutation signatures. Subtle changes in the distribution of immune cells may have different effects on tumor progression [42]. In this study, some wild-type genes showed higher immunocyte infiltration, while others showed lower immunocyte infiltration or no statistical difference (Fig. 7). The stromal score and immune score were based on specific biomarkers associated with stromal and immunocyte infiltration in tumor samples. Stromal and immune scores form the ESTI MATE score, and are negatively correlated with tumor purity [43]. In this study, mutant TP53, APC, KRAS, and PIK3CA had lower stromal score, immune score, ESTIMA TE score and higher tumor purity (Fig. 8). These scores of all genes were consistent with the distribution of immune cells, that is, the mutant genes with lower immunocyte infiltration also had lower immune scores. However, in this study, there was no significant correlation between immunocyte infiltration and RNA expression levels, which needs to be further confirmed and verified in an independent cohort. In addition, the relationship among the expression level of some checkpoints and immunocyte infiltration and TME is also the focus of future research.

In this study, there were two main limitations. First, the availability of data is limited by public resources. The MSI and MSS data that are important to evaluate the CRC patients, cannot get in this study. And some important clinical characteristics were also missing, such as therapeutic regimen, transcriptome sequencing data and expression of immune checkpoints. Second, the small sample number in validation can only prevail the general hypothesis of this study, and the clinical significance of the selected signatures cannot be verified. Therefore, this study need a multi-data analysis and further verification to support the views in the future.

\section{Conclusions}

In conclusion, we have revealed gene mutations and identified 11 gene signatures for the diagnosis, pathological classification, staging and prognosis in CRC, and proved that mutation of most of these signatures significantly reduced their RNA expression, and affected the TME and immunocyte infiltration. This study puts forward further insights into the genotype and therapy of $\mathrm{CRC}$, and is contribute to the personalized diagnosis and treatment.

\section{Abbreviations}

CRC: Colorectal cancer; NGS: Next-generation sequencing; WGS: Whole genome sequencing; OS: Overall survival; PFS: Progression-free survival; MSI: microsatellite instability

\section{Supplementary Information}

The online version contains supplementary material available at https://doi. org/10.1186/s12885-021-08108-9.

\section{Additional file 1.}

Additional file 2.

\section{Acknowledgements}

We would like to thank all of the participants that contributed to this work.

\section{Authors' contributions}

Y Z and HL W: Data curation, Formal analysis, Investigation, Writing-original. D J, Y L, LX F and MY P: Conceptualization, Data curation, Formal analysis, Investigation, Methodology, Validation, Wring-review and editing. CJ T, XW W and JY Z: Data curation, Formal analysis, Investigation, Software, Writingreview and editing. YJ H and PF L: Conceptualization, Data curation, Formal analysis, Funding acquisition, Project administration, Resources, Supervision, Visualization, Writing-original draft, Writing-review and editing. All authors participated in preparing the manuscript and approved the final submitted.

\section{Funding}

This study was supported by Tianjin Science and Technology Plan Project (Grant No. 17ZXMFSY00160). This funding body played no role in the design of the study and collection, analysis, and interpretation of data and in writing the manuscript.

\section{Availability of data and materials}

The datasets used and/or analyzed during the current study are available from the corresponding author on reasonable request.

\section{Declarations}

Ethics approval and consent to participate

The collection of human specimens was approved by the Ethics Committee of Tianjin Medical University Cancer Institute and Hospital, and the written informed consent were obtained from each patient.

\section{Consent for publication}

Not applicable.

\section{Competing interests}

The authors declare that they have no competing interests.

\section{Author details}

${ }^{1}$ Department of Colorectal Oncology, National Clinical Research Center for Cancer, Key Laboratory of Cancer Prevention and Therapy of Tianjin, Tianjin's Clinical Research Center for Cancer, Tianjin Medical University Cancer Institute and Hospital, Tianjin 300060, China. ${ }^{2}$ Department of Oncology, Tianjin Academy of Traditional Chinese Medicine Affiliated Hospital, No.354 Beima Road, Hongqiao District, Tianjin 300120, China. ${ }^{3}$ Department of Medical Oncology, The Fourth Hospital of Hebei Medical University, Shijiazhuang 050000, Hebei, China. ${ }^{4}$ Department of Nursing, Tianjin Cancer Hospital Airport Hospital, Tianjin 300300, China. ${ }^{5}$ Tianjin Marvel Medical Laboratory, Tianjin Marvelbio Technology Co., Ltd, Tianjin 300381, China. ${ }^{6}$ Tianjin Yunquan Intelligent Technology Co., Ltd, Tianjin 300381, China. ${ }^{7}$ Department of Gynecological Oncology, Tianjin Central Hospital of Obstetrics \& Gynecology, No. 156 Nankai Third Road, Nankai District, Tianjin 300100, China.

Received: 22 September 2020 Accepted: 28 March 2021

Published online: 09 April 2021

\section{References}

1. Sung H, Ferlay J, Siegel RL, Laversanne M,Soerjomataram I, Jemal A, et al. Global cancer statistics 2020: GLOBOCAN estimates of incidence and mortality worldwide for 36 cancers in 185 countries. CA: A Cancer J Clin 2020;0:1-41

2. Moghimi-Dehkordi B, Safaee A. An overview of colorectal cancer survival rates and prognosis in Asia. World J Gastrointest Oncol. 2012;4(4):71-5. https://doi.org/10.4251/wjgo.v4.i4.71. 
3. Luo C, Cen S, Ding G, Wu W. Mucinous colorectal adenocarcinoma: clinical pathology and treatment options. Cancer Commun (Lond). 2019;39:13. https://doi.org/10.1186/s40880-019-0361-0.

4. Bi KW, Wei XG, Qin XX, Li B. BTK has potential to be a prognostic factor for lung adenocarcinoma and an Indicator for tumor microenvironment remodeling: a study based on TCGA data mining. Front Oncol. 2020;10:424. https://doi.org/10.3389/fonc.2020.00424.

5. Yi S, Lin S, Li Y, Zhao W, Mills GB, Sahni N. Functional Variomics and network perturbation: connecting genotype to phenotype in Cancer. Nat Rev Genet. 2017;18(7):395-410. https://doi.org/10.1038/nrg.2017.8.

6. Alexandrov LB, Nik-Zainal S, Wedge DC, Aparicio SA, Behjati S, Biankin AV, et al. Signatures of mutational processes in human cancer. Nature. 2013; 500(7463):415-21. https://doi.org/10.1038/nature12477.

7. Kwak MS, Cha JM, Yoon JY, Jeon JW, Shin HP, Chang HJ, et al. Prognostic value of KRAS codon 13 gene mutation for overall survival in colorectal cancer: direct and indirect comparison meta-analysis. Medicine. 2017;96(35): e7882. https://doi.org/10.1097/MD.0000000000007882.

8. Roock WD, Vriendt VD, Normanno N, Ciardiello F, Tejpar S. KRAS, BRAF, PIK3CA, and PTEN mutations: implications for targeted therapies in metastatic colorectal cancer. Lancet Oncol. 2011;12(6):594-603. https://doi. org/10.1016/S1470-2045(10)70209-6.

9. Tian S, Simon I, Moreno V, Roepman P, Tabernero J, Snel M, et al. A combined oncogenic pathway signature of BRAF, KRAS and PI3KCA mutation improves colorectal cancer classification and cetuximab treatment prediction. Gut. 2013;62(4):540-9. https:/doi.org/10.1136/gutjin-2012-302423.

10. Yu J, Wu WK, Li X, He J, Li XX, Ng SS, et al. Novel recurrently mutated genes and a prognostic mutation signature in colorectal cancer. Gut. 2015;64:635-45.

11. Ye ZL, Qiu MZ, Tang T, Wang F, Zhou YX, Lei MJ, et al. Gene mutation profiling in Chinese colorectal cancer patients and its association with clinicopathological characteristics and prognosis. Cancer Med. 2020;9(2): 745-56. https://doi.org/10.1002/cam4.2727.

12. Aiello M, Vella N, Cannavò C, Scalisi A, Spandidos DA, Toffoli G, et al. Role of genetic polymorphisms and mutations in colorectal cancer therapy (review). Mol Med Rep. 2011;4(2):203-8. https://doi.org/10.3892/mmr.2010.408.

13. Bosman FT. Molecular pathology of colorectal cancer. Cytogenet Cell Genet. 1999:86(2):112-7. https://doi.org/10.1159/000015362.

14. Yoshihara K, Shahmoradgoli M, Martínez E, Vegesna R, Kim H, Torres-Garcia $W$, et al. Inferring tumour purity and stromal and immune cell admixture from expression data. Nat Commun. 2013;4(1):2612. https://doi.org/10.1038/ ncomms3612.

15. Hanahan D, Weinberg RA. Hallmarks of cancer: the next generation. Cell. 2011;144(5):646-74. https://doi.org/10.1016/j.cell.2011.02.013.

16. Araghi M, Soeriomataram I, Bardot A, Ferlay J, Cabasag CJ, Morrison DS, et al. Changes in colorectal cancer incidence in seven high-income countries: a population-based study. Lancet Gastroenterol Hepatol. 2019; 4(7):511-8. https://doi.org/10.1016/52468-1253(19)30147-5.

17. Vuik FE, Nieuwenburg SA, Bardou M, Lansdrop-Vogelaar I, Dinis-Ribeiro M, Bento MJ, et al. Increasing incidence of colorectal cancer in young adults in Europe over the last 25 years, 1975-2015. Gut. 2019;0:1-7.

18. Turnbull $C L$, Bacon $A L$, Dunlop MG, Farrington SM. In vitro stability of $A P C$ gene sequences and the influence of DNA repair status. Mutagenesis. 2012; 27(2):233-8. https://doi.org/10.1093/mutage/ger069.

19. lacopetta B. TP53 mutation in colorectal cancer. Hum Mutat. 2003;21(3):2716. https://doi.org/10.1002/humu.10175.

20. Arrington AK, Heinrich EL, Lee W, Duldulao M, Patel S, Sanchez J, et al, Prognostic and predictive roles of KRAS mutation in colorectal Cancer. Int J Mol Sci. 2012;13(12):12153-68. https://doi.org/10.3390/ijms131012153.

21. Wei R, Xiao Y, Song Y, Yuan H, Luo J, Xu W. FAT4 regulates the EMT and autophagy in colorectal cancer cells in part via the PI3K-AKT signaling axis. J Exp Clin Cancer Res. 2019;38(1):112. https://doi.org/10.1186/s13046-019-1043-0.

22. Aisner DL, Nguyen TT, Paskulin DD, Le AT, Haney J, Schulte N, et al. ROS1 and ALK fusions in colorectal Cancer, with evidence of Intratumoral heterogeneity for molecular drivers. Mol Cancer Res. 2014;12(1):111-8. https://doi.org/10.1158/1541-7786.MCR-13-0479-T.

23. Tian X, Sun D, Zhao S, Xiong H, Fang J. Screening of potential diagnostic markers and therapeutic targets against colorectal cancer. Onco Targets Ther. 2015;8:1691-9. https://doi.org/10.2147/OTT.S81621.

24. Torabi K, Miró R, Fernández-Jiménez N, Quintanilla I, Ramos L, Prat E, et al. Patterns of somatic uniparental disomy identify novel tumor suppressor genes in colorectal cancer. Carcinogenesis. 2015;36(10):1103-10. https://doi. org/10.1093/carcin/bgv115.
25. Kowalczyk AE, Krazinski BE, Godlewski J, Kiewiski J, Kwiatkowski P, SliwinskaJewsiewicka A, et al. Expression of the EP300, TP53 and BAX genes in colorectal cancer: correlations with clinicopathological parameters and survival. Oncol Rep. 2017;38(1):201-10. https://doi.org/10.3892/or.2017.5687.

26. Cai J, Feng D, Hu L, Chen H, Yang G, Cai Q, et al. FAT4 functions as a tumour suppressor in gastric cancer by modulating Wnt/ $/$-catenin signalling. $\mathrm{Br} J$ Cancer. 2015;113(12):1720-9. https://doi.org/10.1038/bjc.2015.367.

27. Liao X, Morikawa T, Lochhead P, Imamura Y, Kuchiba A, Yamauchi M, et al. Prognostic role of PIK3CA mutation in colorectal Cancer: cohort study and literature review. Clin Cancer Res. 2012;18(8):2257-68. https://doi.org/10.11 58/1078-0432.CCR-11-2410.

28. Hosseini S, Bananzadeh AM, Salek R, Zare-Bandamiri M, Kermani AT, Mohammadianpanah M. Prognostic significance of mucinous histologic subtype on oncologic outcomes in patients with colorectal Cancer. Ann Coloproctol. 2017;33(2):57-63. https://doi.org/10.3393/ac.2017.33.2.57.

29. Ishaque N, Abba ML, Hauser C, Patil N, Paramasivam N, Huebschmann D, et al. Whole genome sequencing puts forward hypotheses on metastasis evolution and therapy in colorectal cancer. Nat Commun. 2018;9(1):4782-96. https://doi.org/10.1038/s41467-018-07041-z.

30. Hughes D, O'Morain C, Lee N, Kelley L, McMurray H, Latten M, et al. 128 use of a biochip assaying 28 mutations in the KRAS, BRAF, TP53, and APC genes for detection of colorectal neoplasia. Eur J Cancer. 2010;8(5):33-4. https:// doi.org/10.1016/S1359-6349(10)70936-8

31. Smith G, Carey FA, Beattie J, Wikie MJ, Lightfoot TJ, Coxhead J, et al. Mutations in APC, Kirsten-Ras, and P53--alternative genetic pathways to colorectal Cancer. Proc Nati Acad Sci USA. 2002:99(14):9433-8. https://doi. org/10.1073/pnas.122612899

32. Barras D. BRAF mutation in colorectal Cancer: an update. Biomark Cancer. 2015;7(Suppl 1):9-12. https://doi.org/10.4137/BIC.S25248.

33. Kopetz S, Grothey A, Yaeger R, Van Cutsem E, Desai J, Yoshino T, et al. Encorafenib, binimetinib, and cetuximab in BRAF V600E-mutated colorectal cancer. N Engl J Med. 2019;381(17):1632-43. https://doi.org/10.1056/ NEJMoa1908075.

34. Lochhead P, Kuchiba A, Imamura Y, Liao X, Yamauchi M, Nishihara R, et al. Microsatellite instability and BRAF mutation testing in colorectal cancer prognostication. J Natl Cancer Inst. 2013;105(15):1151-6. https://doi.org/10.1 093/jnci/djt173.

35. Wang C, Jette N, Moussienko D, Bebb DG, Lees-Miller SP. ATM-deficient colorectal Cancer cells are sensitive to the PARP inhibitor Olaparib. Transl Oncol. 2017;10(2):190-6. https://doi.org/10.1016/j.tranon.2017.01.007.

36. Geißler AL, Geißler M, Kottmann D, Lutz L, Fichter CD, Fritsch R, et al. ATM mutations and E-cadherin expression define sensitivity to EGFR-targeted therapy in colorectal cancer. Oncotarget. 2017;8(10):17164-90. https://doi. org/10.18632/oncotarget.15211.

37. Tai CJ, Chang CC, Jiang MC, Yeh CM, Su TC, Wu PR. Clinical-pathological correlation of K-Ras mutation and ERK phosphorylation in colorectal cancer. Pol J Pathol. 2012;63(2):93-100.

38. Lupinacci RM, Mello ES, Coelho FF, Kruger JA, Perini MV, Pinheiro RS, et al. Prognostic implication of mucinous histology in resected colorectal cancer liver metastases. Surgery. 2014;155(6):1062-8. https://doi.org/10.1016/j.surg.2 014.01.011.

39. Pietrantonio F, Di Nicolantonio F, Schrock AB, Lee J, Tejpar S, Sartore-Bianchi A, et al. ALK, ROS1, and NTRK rearrangements in metastatic colorectal Cancer. J Nati Cancer Inst. 2017;109.

40. Kato S, lida S, Higuchi T, Ishikawa T, Takagi Y, Yasuno M, et al. PIK3CA mutation is predictive of poor survival in patients with colorectal cancer. Int J Cancer. 2007;121(8):1771-8. https://doi.org/10.1002/ijc.22890.

41. Jia P, Zhao Z. Impacts of somatic mutations on gene expression: an association perspective. Brief Bioinform. 2017;18(3):413-25. https://doi.org/1 0.1093/bib/bbw037.

42. Pan S, Zhan Y, Chen X, Wu B, Liu B. Bladder cancer exhibiting high immunocyte infiltration shows the lowest response rate to immune checkpoint inhibitors. Front Oncol. 2019;9:1101. https://doi.org/10.3389/ fonc.2019.01101.

43. Luo Q, Vögeli TA. A methylation-based reclassification of bladder Cancer based on immune cell genes. Cancers (Basel). 2020;12(10):3054. https://doi. org/10.3390/cancers12103054.

\section{Publisher's Note}

Springer Nature remains neutral with regard to jurisdictional claims in published maps and institutional affiliations. 